\title{
Combination of Juzentaihoto and chemotherapy improves the prognosis of patients with postoperative recurrence of non-small cell lung cancer
}

\author{
HIDEKI KAWAI and YOSHITARO SAITO \\ Department of Thoracic Surgery, Akita Red Cross Hospital, Akita 010-1495, Japan
}

Received January 20, 2020; Accepted May 26, 2020

DOI: $10.3892 / \mathrm{mco} .2020 .2083$

\begin{abstract}
Kampo medicines have been used to reduce chemotherapy-induced adverse events. However, whether Kampo medicine can improve the prognosis of cancer remains unclear. The present study aimed to clarify the effect of Juzentaihoto (TJ-48) on patients with postoperative recurrence of non-small cell lung cancer. In total, 45 patients with postoperative recurrent non-small cell lung cancer scheduled for first-line chemotherapy were enrolled in the present study. Differences in progression-free survival between the chemotherapy combined with TJ-48 and chemotherapy only groups were analyzed. Body weight change and prognostic nutritional index were also evaluated to examine whether these factors were influenced by TJ-48 administration. Multivariate analysis was performed to detect independent prognostic factors. A significant increase was observed in progression-free survival in the chemotherapy plus TJ-48 group compared with in the chemotherapy alone group $(\mathrm{P}<0.001)$. Significant decreases in body weight and prognostic nutritional index score were observed in the chemotherapy alone group $(\mathrm{P}<0.01$ and $\mathrm{P}<0.05$, respectively); however, these decreases were not observed in the chemotherapy plus TJ-48 group. Multivariate analysis revealed that TJ-48 administration with chemotherapy was an independent prognostic factor. In conclusion, TJ-48 combined with chemotherapy may improve the progression-free survival of patients with postoperative recurrence of non-small cell lung cancer by preventing nutritional disorders.
\end{abstract}

\section{Introduction}

Lung cancer is known for its poor prognosis, and chemotherapy is mainly conducted as therapy in patients with postoperative recurrence. Therapeutic options have varied with the increase

Correspondence to: Dr Hideki Kawai, Department of Thoracic Surgery, Akita Red Cross Hospital, 222-1 Naeshirosawa, Saruta, Kamikitate, Akita 010-1495, Japan

E-mail: hideki_kawai@akita-med.jrc.or.jp

Key words: Juzentaihoto, non-small cell lung cancer, chemotherapy, prognostic nutritional index, postoperative recurrence in the development of novel anticancer drugs (1). However, this has not led to a dramatic improvement in the survival rate, particularly for patients who are not eligible for molecular targeted therapy. One of the problems of chemotherapy is adverse drug reactions, which damage the host's body and weaken their immune system. As a result, the therapy is difficult to safely continue and leads to cancer cachexia. In particular, attention should be paid when chemotherapy is applied for recurrence after lung cancer surgery because it may cause fatal side effects due to decreased physical fitness, such as decreased pulmonary function. To improve the success of postoperative recurrent lung cancer treatment, managing chemotherapy-induced adverse drug reactions and immune function is necessary.

Kampo medicine has been used as hozai (supportive drug) in Japan for the reduction of anticancer drug-induced adverse reactions (2-5). Recently, there have been some reports that Kampo medicine enhances immune function and anticancer action and that its combined use with chemotherapy boosts the therapeutic effect of anticancer drugs (6-8). In a pharmacological study investigating the enhancement of the anticancer effect or improvement of the immune system, ginsenosides in ginseng, one of the components used in Kampo medicine, demonstrated a decrease in adverse drug reactions of anticancer drugs and reinforced the therapeutic effect (9-11).

Among several Kampo medicines, Juzentaihoto (TJ-48) is used to improve the following symptoms in people with poor physical strength: Physical strength decline after illness or surgery, fatigue/malaise, loss of appetite, night sweat, coldness of hands and feet, and anemia. In studies on the basic mechanism of TJ-48's effect, its effects have been reported through experimental models such as the stimulation of natural killer activity, anticancer cytokine production, or enhancement of blood synthesis (12-15). However, there is no appropriate method in conventional clinical practice to evaluate the actual effect of TJ-48 for improving the prognosis of patients with cancer since it has been considered as hozai and is used with other anticancer drugs (7). To solve this problem, we focused on the relationship between cancer chemotherapy and the effect of TJ-48 by evaluating nutritional status.

In this study, to investigate the effect of $\mathrm{TJ}-48$ on patients with chemotherapy, we conducted prospective clinical research 
to assess the effects of chemotherapy with or without TJ-48 in patients with postoperative recurrent non-small cell lung cancer (NSCLC).

\section{Patients and methods}

Patients. This prospective study was conducted with the approval of the Human Ethics Committee of Akita Red Cross Hospital (approval no. H26-7). Written informed consent was obtained from all patients before study enrollment. A total of 45 patients with postoperative recurrent NSCLC scheduled for first-line chemotherapy were enrolled in this study. There are 13 EGFR mutation positive patients included in this study. Table I shows the patients' characteristics. Of the 45 patients, 23 were administered TJ-48 (Chemo + TJ-48 group) and 22 received chemotherapy alone (Chemo alone group). Progression-free survival (PFS) was compared between these two groups. PFS was defined as the period from the start of chemotherapy to exacerbation, i.e., increase in neoplasm, novel metastasis, and the rise in tumor markers. Tumor size was evaluated with computed tomography using the Response Criteria in Solid Tumors version 1.1 (16). The patients were examined by blood analysis and chest X-ray every 2 weeks during chemotherapy. The patients were also examined with computed tomography 1 month after chemotherapy was started.

TJ-48. The patients received TJ-48 t.i.d.(7.5 g/day) before each meal for 2 weeks before the start of chemotherapy until they were diagnosed with disease progression. The components containing TJ-48 are shown in Table II. To investigate the influence of TJ-48 on immune status, serum albumin, and total lymphocytes were measured by peripheral blood examination at the time of the start of TJ-48 administration, chemotherapy, and 2 months after chemotherapy.

Statistical analysis. A univariate analysis regarding the patients' characteristics was performed by unpaired t-test using the Fisher's exact test for sex, surgical procedure, and $\mathrm{G}$ test for other factors. We analyzed the prognostic difference between the two groups using the Kaplan-Meier method and log-rank tests. We also evaluated the change in body weight by unpaired t-test (Welch t-test), prognostic nutrition index (PNI) calculated by serum albumin, and total lymphocytes using a paired t-test, with $\mathrm{P}<0.05$ considered significant. To assess the body weight loss ratio, the ratio of body weight within 1 month before and 2 months after chemotherapy was used. The body weight loss ratio was calculated as follows: 1-(postoperative body weight/preoperative body weight). When receiver operating characteristic curves were drawn for the PNI and body weight loss ratio, the cutoff values were 46.7 and 3.0, respectively. PNI was calculated as $10 \mathrm{x}$ serum albumin $(\mathrm{g} / \mathrm{dl})+0.005 \mathrm{x}$ total lymphocyte $\left(/ \mathrm{mm}^{3}\right)$, as described previously (17). A multivariate analysis was performed using the Cox proportional hazard model. Statistical analyses were performed using JMP IN 10.0.2 software program (SAS Institute).

\section{Results}

Effect of TJ-48 on chemotherapy. We compared the PFS rate between the Chemo + TJ-48 and Chemo alone groups (Fig. 1).
We found that the PFS rate in the Chemo + TJ-48 group was significantly higher than that in the Chemo alone group $(\mathrm{P}<0.001)$. There were 13 EGFR mutation positive patients in this study. In cases without mutation, the average time to progression was 355.8 days with TJ-48, which was significantly longer than 89.2 days without TJ-48. On the other hand, in cases with EGFR mutation, there was an also significant prolongation with 684.3 days with TJ-48 against 361.8 days without TJ-48 (data not shown).

TJ-48 reduces adverse events caused by chemotherapy. We observed various grades of adverse events during chemotherapy (Table I). The following courses of chemotherapy were administered: Platinum doublet (five cases of carboplatin + pemetrexed, three cases of cisplatin + pemetrexed, three cases of carboplatin + paclitaxel, and one case of carboplatin + gemcitabine), single agents (20 cases of pemetrexed, five cases of docetaxel, three cases of Tegafur, and two cases of S-1), and EGFR-TKI (two cases of gefitinib and one case of erlotinib). Adverse events were observed in nine $(39.1 \%)$ cases in the Chemo + TJ-48 group and $18(81.8 \%)$ cases in the Chemo alone group. A statistically significant difference was noted between the two groups $(\mathrm{P}<0.01)$. Furthermore, adverse events of $\geq \mathrm{G} 3$ were observed in only one $(4.3 \%)$ case in the Chemo + TJ-48 group, whereas six (27.3\%) cases occurred in the Chemo alone group.

Effect of TJ-48 on nutritional status. To examine whether TJ-48 influenced nutritional status, we measured the body weight change and PNI before and after therapy. We found that statistically significant body weight loss was observed in the Chemo alone group compared with the Chemo + TJ-48 group $(\mathrm{P}<0.01$; Fig. 2). The PNI score was statistically significantly decreased in the Chemo alone group $(\mathrm{P}<0.05)$, whereas there was no change in the Chemo + TJ-48 group ( $\mathrm{P}=0.11$; Fig. 3 ).

Multivariate analysis for PFS. We next performed univariate and multivariate analyses for PFS; the results are shown in Table III. In the univariate analysis for PFS, female (HR, 0.45; 95\% CI, 0.23-0.85), TJ-48 administration (HR, 0.36; 95\% CI, 0.18-0.65), PNI on pretreatment (HR, 0.48; 95\% CI, 0.25-0.92), and body weight loss ratio (HR, 2.74; 95\% CI, 1.40-5.34) were statistically significant. In the multivariate analysis for these factors, only TJ-48 administration was significantly correlated with PFS (HR, 0.47; 95\% CI, 0.23-0.93).

\section{Discussion}

Surgical outcomes for NSCLC are relatively positive in the early stages; however, in advanced lung cancer such as stage III, the prognosis remains poor (18). In advanced cancer, the probability of recurrence after surgery is extremely high, so a treatment strategy after postoperative recurrence is important for improving prognosis. Regarding the use of anticancer drugs in the surgical field, lung cancer treatment includes preoperative chemotherapy, postoperative adjuvant chemotherapy for advanced cancer, and treatment of postoperative recurrence. Postoperative recurrence treatment, in particular, is more likely to cause adverse events compared with other situations because of a decrease of postoperative lung function and loss of physical fitness due to recurrence. 
Table I. Characteristics of 45 patients with postoperative recurrence of non-small cell lung cancer.

\begin{tabular}{|c|c|c|c|}
\hline Variable & Chemo + TJ-48 $(n=23)$ & Chemo alone $(n=22)$ & P-value \\
\hline Age (years; median, range) & $65(38-87)$ & $68(42-80)$ & 0.52 \\
\hline Sex (male/female) & $11 / 12$ & $19 / 3$ & 0.01 \\
\hline Histologic type (Ad/Scc/Large) & $20 / 2 / 1$ & $15 / 5 / 2$ & 0.31 \\
\hline Chemotherapy (platinum doublet/single/molecular) & $6 / 15 / 2$ & $6 / 15 / 1$ & 0.85 \\
\hline Adverse events (None/<G2/>G3) & $14 / 8 / 1$ & $4 / 12 / 6$ & 0.01 \\
\hline Surgical procedure (VATS/Standard thoracotomy) & $21 / 2$ & $16 / 6$ & 0.13 \\
\hline Type of surgery (Lobe/Pneumo/Seg) & $23 / 0 / 0$ & $18 / 2 / 2$ & 0.05 \\
\hline Pathological stage at surgery (I/II/III/IV) & $5 / 9 / 7 / 2$ & $7 / 5 / 10 / 0$ & 0.19 \\
\hline Metastatic organ (Brain/Lung/Liver/Bone/Others ${ }^{\mathrm{a}}$ ) & $4 / 11 / 1 / 1 / 6$ & $5 / 6 / 3 / 2 / 6$ & 0.56 \\
\hline
\end{tabular}

${ }^{a}$ Others included: Adrenal gland, pleura and mediastinal lymph node. Ad, adenocarcinoma; Scc, squamous cell carcinoma; single, single agents; molecular, molecular target; VATS, video-assisted thoracic surgery; Lobe/Pneumo/Seg, Lobectomy/Pneumonectomy/Segmentectomy; Chemo, chemotherapy; TJ-48, Juzentaihoto; VATS, video-assisted thoracic surgery.

Table II. List of ingredients in Juzentaihoto (TJ-48) extract granules (7.5 g).

Extract: Plant species

Component ratio $(\%)$

Astragalus Root: Astragalus membranaceus Bunge

Cinnamon Bark: Cinnamomum cassia Blume

10.5

Rehmannia Root: Rehmannia glutiosa Libosch. Var. purpurea Makino

10.5

Peony Root: Paeonia lactiflora Pallas

10.5

Cnidium Rhizome: Cnidium officinale Makino

10.5

Atractylodes Lancea Rhizome: Atractylodes lancea De Candole 10.5

Japanese Angelica Root: Angelica acutiloba Kitagawa 10.5

Ginseng: Panax ginseng C.A. Mey. 10.5

Poria Sclerotium: Poria cocos Wolf 10.5

Glycyrrhiza: Glycyrrhiza uralensis Fisher $\quad 5.5$

In $7.5 \mathrm{~g}$ of this product, $5.0 \mathrm{~g}$ dry extract of the mixed crude drug was included at the indicated ratio.

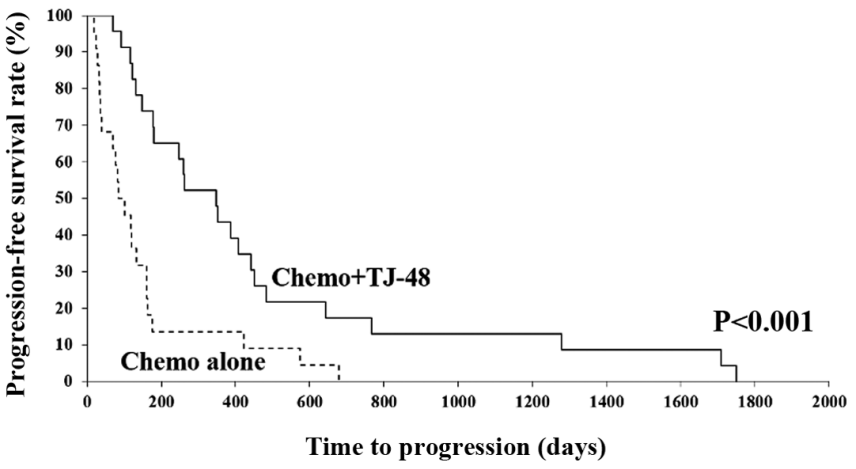

Figure 1. Kaplan-Meier analysis regarding chemo with TJ-48 (Chemo + TJ-48) or without TJ-48 (Chemo alone group). The P-value was calculated using a log-rank test. Chemo, chemotherapy; TJ-48, Juzentaihoto.

Conversely, with the advance of anticancer drugs, which is represented by the emergence of molecular target drugs and immune checkpoint inhibitors, the diversification of chemotherapy is progressing (19-22). In chemotherapy, both

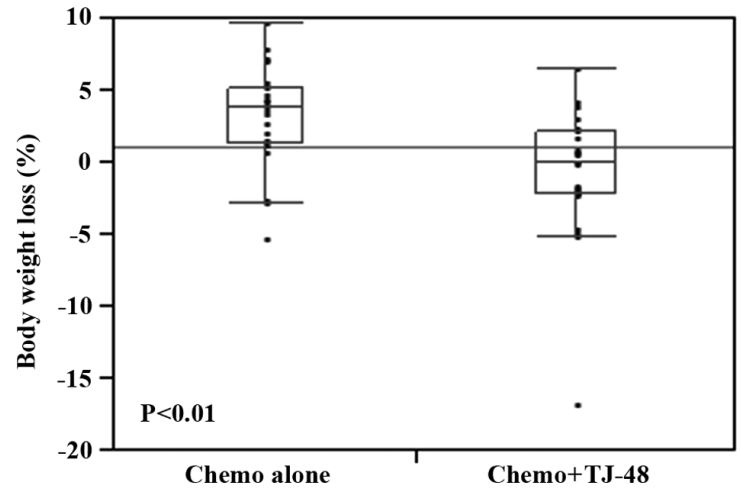

Figure 2. Comparison of body weight changes between the two groups. The $\mathrm{P}$-value was determined using Welch t test. Chemo, chemotherapy; TJ-48, Juzentaihoto.

the drug effects and the appearance of adverse events are important problems. Conventional cytotoxic anticancer drugs have several adverse events such as gastrointestinal symptoms, blood toxicity, neurotoxicity, and interstitial pneumonia. 
Table III. Univariate and multivariate analysis for progression-free survival.

\begin{tabular}{|c|c|c|c|c|c|c|}
\hline \multirow[b]{2}{*}{ Variable } & \multicolumn{3}{|c|}{ Univariate analysis } & \multicolumn{3}{|c|}{ Multivariate analysis } \\
\hline & Hazard ratio & $95 \% \mathrm{CI}$ & P-value & Hazard ratio & $95 \% \mathrm{CI}$ & P-value \\
\hline Female & 0.45 & $0.23-0.85$ & $0.01^{\mathrm{a}}$ & 0.57 & $0.27-1.14$ & 0.11 \\
\hline Adenocarcinoma & 0.57 & $0.28-1.23$ & 0.14 & & & \\
\hline$>70$ years & 1.37 & $0.70-2.57$ & 0.35 & & & \\
\hline Juzentaihoto administration & 0.36 & $0.18-0.65$ & $<0.01^{\mathrm{a}}$ & 0.47 & $0.23-0.93$ & $0.03^{\mathrm{a}}$ \\
\hline Pretreatment PNI $\geq 46.7^{\mathrm{b}}$ & 0.48 & $0.25-0.92$ & $0.03^{\mathrm{a}}$ & 0.71 & $0.36-1.39$ & 0.31 \\
\hline Body weight loss ratio $\geq 3.0^{\mathrm{b}}$ & 2.74 & $1.40-5.34$ & $<0.01^{\mathrm{a}}$ & 1.69 & $0.80-3.52$ & 0.17 \\
\hline
\end{tabular}

${ }^{\mathrm{a}} \mathrm{P}<0.05$ was considered to indicate a statistically significant difference. ${ }^{\mathrm{b}}$ For PNI and body weight loss ratio, the cutoff values were obtained by receiver operating characteristic curves as 46.7 and 3.0, respectively. PNI, prognostic nutrition index; CI, confidence interval.

A

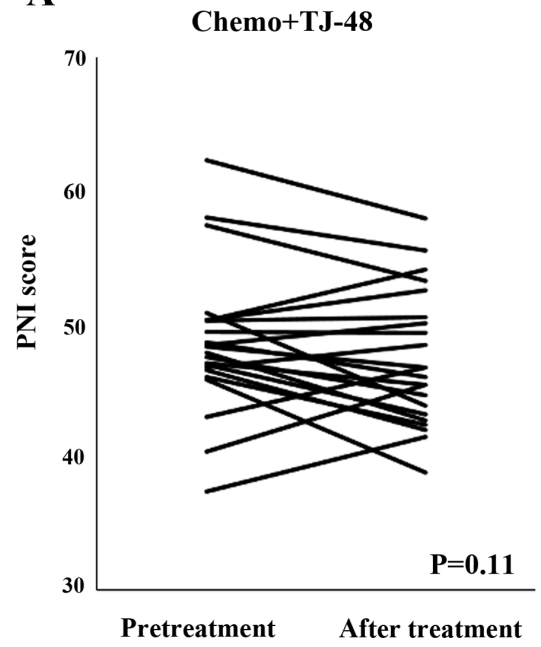

B

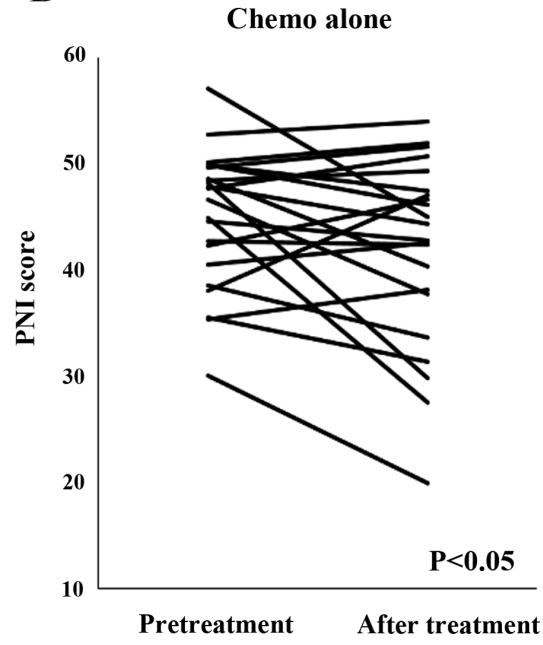

Figure 3. Comparison of the PNI score before and after treatment. PNI was evaluated 2 weeks before treatment and 2 months after treatment. The P-value was obtained using a paired t-test. (A) Chemo + TJ-48. (B) Chemo alone. Chemo, chemotherapy; PNI, prognostic nutrition index; TJ-48, Juzentaihoto.

Furthermore, the development of interstitial pneumonia is an adverse event that affects the outcome of molecular targeted drugs and immune checkpoint inhibitors $(23,24)$.

Recently, in response to the occurrence of adverse events, steroids have been used for immune-related adverse events with immune checkpoint inhibitors $(25,26)$. Since steroids have both an anti-inflammatory effect and an immunosuppressive effect, they may be negative for cancer treatment. In addition, when side effects appear, quality of life decreases, leading to a decline in immunity and a worse prognosis. Two contradictory elements are necessary to improve prognosis: Not reducing physical fitness and using effective anticancer drugs; however, adverse events and their treatment have a negative impact on the former factor. Therefore, it is important to select a treatment suitable for physical strength in the treatment strategy, and in lung cancer chemotherapy, treatment with a single agent or two agents has conventionally been performed.

However, multidrug therapy is recommended as a recent treatment for unresectable NSCLC $(27,28)$. As is well known, there are no items focused on postoperative recurrence cases in the lung cancer clinical practice guidelines, and treatment is selected according to the unresectable cancer. However, it is difficult to include recurrence cases after surgery as unresectable cancer because the patient's condition is very different, as described above. It is important to continue treatment without difficulty while maintaining QOL in the treatment strategy for postoperative recurrence.

In this study, the role of Kampo medicines used as a side effect countermeasure was examined from different angles, and TJ-48 combination chemotherapy was shown to be effective in improving prognosis. This result suggests the possibility of a new Kampo treatment.

In recent years, studies using nutritional status to predict the prognosis of lung cancer treatment have been reported to be useful in predicting prognosis (29-38). For example, there are studies on the correlation between body weight loss rate and prognosis and on preoperative body mass index or prealbumin levels for predicting prognosis (36-38). There are also reports of nutritional indicators; among them, PNI is reported to be a simple and useful indicator (29-35). However, no reports have shown that prognosis after recurrence has been improved by preventing a decrease in physical fitness or malnutrition. Some immunonutrition studies have reported that preoperative immunonutrient administration contributes 
to the suppression of postoperative complications, but there is no report on the long-term prognostic improvement (39).

This study showed for the first time that the decrease in physical strength due to side effects of anticancer drugs exacerbates prognosis and that TJ-48 prevents malnutrition and contributes to the improvement of prognosis. Currently, the treatment strategy for postoperative recurrence is debatable, and TJ-48 combination therapy is considered an effective choice to improve prognosis.

Kampo has not been actively used thus far partially because there was a misconception that it takes time to be effective. In addition, the effect was not evaluated, and it was used indiscriminately. Furthermore, there are prescription indications specific to Kampo, represented by 'sho,' which is one of the reasons why general clinicians hesitate to prescribe it. The 'sho' is, in an easy-to-understand manner, 'a thing that represents the person's condition (constitutional, physical strength, resistance, and symptom differences).' Even if Kampo, which is said to have few side effects, does not meet the 'sho', there may be an adverse event; however, even if it is not possible to evaluate the 'sho', it can be used appropriately for the indications, taking into account age and performance status. Therefore, prescriptions that match the constitution will likely be possible. To prevent the abuse of Kampo, it is important to observe the condition after prescribing and not to prescribe it without proper evaluation. Although no effective evaluation of the effects of Kampo medicine on cancer-bearing patients has been reported thus far, the nutritional evaluation shown in this study may be useful. Above all, weight change should be evaluated because it is simple and possible at home.

There is some limitation for this study. First, this study demonstrated that TJ-48 improves prognosis by preventing nutritional deterioration by decreasing adverse events of anti-cancer drugs. Unfortunately, because of clinical study, we did not perform any experimental examination such as in vitro mechanistic analysis. We are very interested in the direct anticancer effects of TJ-48, but I look forward to future research. Second, the presence or absence of EGFR mutation is an important factor in lung cancer treatment. There were 13 EGFR mutation positive patients in this study. In cases without mutation, the average time to progression was 355.8 days with TJ-48, which was significantly longer than 89.2 days without TJ-48. On the other hand, in cases with EGFR mutation, there was an also significant prolongation with 684.3 days with TJ-48 against 361.8 days without TJ-48. However, in this study, the sample size is small and the statistical power of the sub-analysis is weak, so we think that it is necessary to further increase the sample size to analyze in detail in the future.

In conclusion, we demonstrated that in the severe prognosis of postoperative recurrent lung cancer, the use of TJ-48 in combination with anticancer drug treatment reduced the incidence of side effects, and as a result, a decrease in physical fitness was prevented, thereby improving the prognosis using a nutritional assessment and multivariate analysis. The use of TJ-48 can be an effective means to maximize the effects of chemotherapy and contribute to improved prognosis.

\section{Acknowledgements}

Not applicable.

\section{Funding}

No funding was received.

\section{Availability of data and materials}

All data generated or analyzed during this study are included in this published article.

\section{Authors' contributions}

HK made substantial contributions to conception and design, acquisition of data, and writing of the manuscript. YS analyzed and interpreted the patient data. All authors read and approved the final manuscript.

\section{Ethics approval and consent to participate}

This prospective study was conducted with the approval of the Human Ethics Committee of Akita Red Cross Hospital (approval no. H26-7). Written informed consent was obtained from all patients prior to study enrollment.

\section{Patient consent for publication}

Identifying information was not be included in the manuscript and written informed consent for the publication of any associated data was obtained from all patients.

\section{Competing interests}

The authors declare that they have no competing interests.

\section{References}

1. Yano T, Okamoto T, Fukuyama S and Maehara Y: Therapeutic strategy for postoperative recurrence in patients with non-small cell lung cancer. World J Clin Oncol 5: 1048-1054, 2014.

2. Ohnishi S and Takeda H: Herbal medicines for the treatment of cancer chemotherapy-induced side effects. Front Pharmacol 6: $14,2015$.

3. Inoue T, Takagi H, Owada $\mathrm{Y}$, Watanabe $\mathrm{Y}$, Yamaura $\mathrm{T}$, Fukuhara M, Muto S, Okabe N, Matsumura Y, Hasegawa T, et al: The efficacy of the Kampo medicine rikkunshito for chemotherapy-induced anorexia (RICH trial): Study protocol for a randomized controlled trial. Trials 18: 485, 2017.

4. Mori K, Kondo T, Kamiyama Y, Kano Y and Tominaga K: Preventive effect of Kampo medicine (Hangeshashin-to) against irinotecan-induced diarrhea in advanced non-small-cell lung cancer. Cancer Chemother Pharmacol 51: 403-406, 2003.

5. Ichiki M, Wataya H, Yamada K, Tsuruta N, Takeoka H, Okayama Y, Sasaki J and Hoshino T: Preventive effect of kampo medicine (hangeshashin-to, TJ-14) plus minocycline against afatinib-induced diarrhea and skin rash in patients with non-small cell lung cancer. OncoTargets and Therapy 10: 5107-5113, 2017.

6. Ikemoto T, Shimada M, Iwahashi S, Saito Y, Kanamoto M, Mori H, Morine Y, Imura S and Utsunomiya T: Changes of immunological parameters with administration of Japanese Kampo medicine (Juzen-Taihoto/TJ-48) in patients with advanced pancreatic cancer. Int J Clin Oncol 19: 81-86, 2014.

7. Amitani M, Amitani H, Sloan RA, Suzuki H, Sameshima N, Asakawa A, Nerome Y, Owaki T, Inui A and Hoshino E: The translational aspect of complementary and alternative medicine for cancer with particular emphasis on Kampo. Front Pharmacol 6: 150, 2015

8. Takeno N, Inujima A, Shinohara K, Yamada M, Shibahara N, Sakurai H, Saiki I and Koizumi K: Immune adjuvant effect of Juzentaihoto, a Japanese traditional herbal medicine, on tumor vaccine therapy in a mouse model. Int J Oncol 47: 2115-2122, 2015. 
9. Li QF, Shi SL, Liu QR, Tang J, Song J and Liang Y: Anticancer effects of ginsenoside Rg1, cinnamic acid, and tanshinone IIA in osteosarcoma MG-63 cells: Nuclear matrix downregulation and cytoplasmic trafficking of nucleophosmin. Int J Biochem Cell Biol 40: 1918-1929, 2008 .

10. He BC, Gao JL, Luo X, Luo J, Shen J, Wang L, Zhou Q, Wang YT, Luu HH, Haydon RC, et al: Ginsenoside Rg3 inhibits colorectal tumor growth through the down-regulation of Wnt/ $\beta$-catenin signaling. Int J Oncol 38: 437-445, 2011.

11. Li L, Wang Y, Qi B, Yuan D, Dong S, Guo D, Zhang C and Yu M: Suppression of PMA-induced tumor cell invasion and migration by ginsenoside Rg1 via the inhibition of NF- $\kappa B$-dependent MMP-9 expression. Oncol Rep 32: 1779-1786, 2014.

12. Kamiyama $\mathrm{H}$, Takano S, Ishikawa E, Tsuboi K and Matsumura A: Anti-angiogenic and immunomodulatory effect of the herbal medicine 'Juzen-taiho-to' on malignant glioma. Biol Pharm Bull 28: 2111-2116, 2005.

13. Matsuda T, Maekawa K, Asano K and Hisamitsu T: Suppressive effect of Juzen-Taiho-To on lung metastasis of B16 melanoma cells in vivo. Evid Based Complement Alternat Med 2011: 743153, 2011.

14. Saiki I: A Kampo medicine 'Juzen-taiho-to'-prevention of malignant progression and metastasis of tumor cells and the mechanism of action. Biol Pharm Bull 23: 677-688, 2000.

15. Onishi Y, Yamaura T, Tauchi K, Sakamoto T, Tsukada K, Nunome S, Komatsu Y and Saiki I: Expression of the anti-metastatic effect induced by Juzen-taiho-to is based on the content of Shimotsu-to constituents. Biol Pharm Bull 21: 761-765, 1998.

16. Eisenhauer EA, Therasse P, Bogaerts J, Schwartz LH, Sargent D, Ford R, Dancey J, Arbuck S, Gwyther S, Mooney M, et al: New response evaluation criteria in solid tumours: Revised RECIST guideline (version 1.1). Eur J Cancer 45: 228-247, 2009.

17. Shirakabe A, Hata N, Kobayashi N, Okazaki H, Matsushita M Shibata Y, Nishigoori S, Uchiyama S, Asai K and Shimizu W: The prognostic impact of malnutrition in patients with severely decompensated acute heart failure, as assessed using the Prognostic Nutritional Index (PNI) and Controlling Nutritional Status (CONUT) score. Heart Vessels 33: 134-144, 2018.

18. Sawabata N, Asamura H, Goya T, Mori M, Nakanishi Y, Eguchi K, Koshiishi Y, Okumura M, Miyaoka E and Fujii Y; Japanese Joint Committee for Lung Cancer Registry: Japanese Lung Cancer Registry Study: First prospective enrollment of a large number of surgical and nonsurgical cases in 2002. J Thorac Oncol 5: 1369-1375, 2010.

19. Hong W, Wu Q, Zhang J and Zhou Y: Prognostic value of EGFR19-del and 21-L858R mutations in patients with non-small cell lung cancer. Oncol Lett 18: 3887-3895, 2019.

20. Akamatsu H, Katakami N, Okamoto I, kato T, Kim YH Imamura F, Shinkai M, Hodge RA, Uchida $\mathrm{H}$ and Hida $\mathrm{T}$ : Osimertinib in Japanese patientswith EGFR T790M mutation-positive advanced non-small-cell lung cancer: AURA3 trial Cancer Sci 109: 1930-1938, 2018.

21. Shukuya T, Mori K, Amann JM, Bertino EM, Otterson GA, Shields PG, Morita S and Carbone DP: Relationship between overall survival and response or progression free survival in advanced non-small cell lung cancer patients treated with anti-PD-1/PD-L1 antibodies. J Thorac Oncol 11: 1927-1939, 2016.

22. Passiglia F, Galvano A, Rizzo S, Incorvaia L, Listi A, Bazan V and Russo A: Looking for the best immune-checkpoint inhibitor in pre-treated NSCLC patients: An indirect comparison between nivolumab, pembrolizumab and atezolizumab. Int J Cancer 142 $1277-1284,2018$

23. Sakurada T, Kakiuchi S, Tajima S, Horinouchi Y, Okada N, Nishisako $H$, Nakamura $T$, Teraoka $K$, Kawazoe $K$, Yanagawa $\mathrm{H}$, et al: Characteristics of and risk factors for interstitial lung disease induced by chemotherapy for lung cancer. Ann Pharmacother 49: 398-404, 2015.

24. Kalisz KR, Ramaiya NH, Laukamp KR and Gupta A: Immune checkpoint inhibitor therapy-related pneumonitis: Pattern and management. Radiographics 39: 1923-1937, 2019.
25. Michot JM, Bigenwald C, Champiat S, Collins M, Carbonnel F, Postel-Vinay S, Berdelou A, Varga A, Bahleda R, Hollebecque A, et al: Immune-related adverse events with immune checkpoint blockade: A comprehensive review. Eur J Cancer 54: 139-148, 2016.

26. Brahmer JR, Lacchetti C, Schneider BJ, Atkins MB, Brassil KJ, Caterino JM, Chau I, Ernstoff MS, Gardner JM, Ginex P, et al: Management of immune-related adverse events in patients treated with immune checkpoint inhibitor therapy: American Society of Clinical Oncology clinical practice guideline. J Clin Oncol 36: 1714-1768, 2018.

27. Gandhi L, Rodríguez-Abreu D, Gadgeel S, Esteban E, Felip E, De Angelis F, Domine M, Clingan P, Hochmair MJ, Powell SF, et al: Pembrolizumab plus chemotherapy in metastatic non-small-cell lung cancer. N Engl J Med 378: 2078-2092, 2018.

28. Socinski MA, Jotte RM, Cappuzzo F, Orlandi F, Stroyakovskiy D, Nogami N, Rodríguez-Abreu D, Moro-Sibilot D, Thomas CA, Barlesi F, et al: Atezolizumab for first-line treatment of metastatic nonsquamous NSCLC. N Engl J Med 378: 2288-2301, 2018.

29. Mori S, Usami N, Fukumoto K, Mizuno T, Kuroda H, Sakakura N, Yokoi K and Sakao Y: The significance of the prognostic nutritional index in patients with completely resected non-small cell lung cancer. PLoS One 10: e0136897, 2015.

30. Shimizu K, Okita R, Saisho S, Maeda A, Nojima Y and Nakata M: Preoperative neutrophil/lymphocyte ratio and prognostic nutritional index predict survival in patients with non-small cell lung cancer. World J Surg Oncol 13: 291, 2015.

31. Shoji F, Morodomi Y, Akamine T, Takamori S, Katsura M, Takada K, Suzuki Y, Fujishita T, Okamoto T and Maehara Y: Predictive impact for postoperative recurrence using the preoperative prognostic nutritional index in pathological stage I non-small cell lung cancer. Lung Cancer 98: 15-21, 2016.

32. Sheng J, Yang YP, Ma YX, Qin T, Hu ZH, Hong SD, Zhou T, Huang Y, Zhao HY and Zhang L: Low prognostic nutritional index correlates with worse survival in patients with advanced NSCLC following EGFR-TKIs. PLoS One 11: e0147226, 2016.

33. Okada S, Shimada J, Kato D, Tsunezuka H, Teramukai S and Inoue M: Clinical significance of prognostic nutritional index after surgical treatment in lung cancer. Ann Thorac Surg 104: 296-302, 2017.

34. Hu Y, Shen J, Liu R, Feng Z, Zhang C, Ling L and Chen L: Prognostic value of pretreatment prognostic nutritional index in non-small cell lung cancer: A systematic review and meta-analysis. Int J Biol Markers 33: 372-378, 2018.

35. Ramos R, Nadal E, Peiró I, Masuet-Aumatell C, Macia I, Rivas F, Rosado G, Rodriguez P, Ureña A, Padrones, et al: Preoperative nutritional status assessment predicts postoperative outcomes in patients with surgically resected non-small cell lung cancer. Eur J Surg Oncol 44: 1419-1424, 2018.

36. Kawai $\mathrm{H}$ and Ota $\mathrm{H}$ : Low perioperative prealbumin predicts early recurrence after curative pulmonary resection for non-small-cell lung cancer. World J Surg 26: 2853-2857, 2012.

37. Nakagawa T, Toyozaki T, Chiba N, Ueda Y and Gotoh M: Prognostic value of body mass index and change in body weight in postoperative outcomes of lung cancer surgery. Interact CardioVasc Thorac Surg 23: 560-566, 2016.

38. Kawai H, Saito Y and Suzuki Y: Gender differences in the correlation between prognosis and postoperative weight loss in patients with non-small cell lung cancer. Interact CardioVasc Thorac Surg 25: 272-277, 2017.

39. Braga M, Gianotti L, Vignali A and Carlo VD: Preoperative oral arginine and n-3 fatty acid supplementation improves the imminometabolic host response and outcome after colorectal resection for cancer. Surgery 132: 805-814, 2002. 